\title{
Lymphocytes populations in appendiceal lavage fluid predictive of IBD-related inflammation
}

\begin{abstract}
Background: The objective of this study was to evaluate if appendiceal lymphocyte infiltration can be determined by analysis of lavage fluid and whether discrimination was possible between both types of IBD, acute appendicitis, and controls.

Methods: The appendix of 15 ulcerative colitis (5 in remission), 9 Crohn's disease, 7 acute appendicitis, and 10 non-inflammatory controls were surgically removed and flushed with 2cc of phosphate buffered saline. CD4+ and CD8+ lymphocytes in the lavage fluid were determined by FACS analysis. Mucosal and transmural inflammation was graded according to the validated Geboes score. Results were correlated to clinical disease activity.

Results: The appendices of UC patients showed mucosal inflammation in $80 \%(12 / 15$, Geboes score $\geq 2$ ) despite a macroscopically normal appearance. A high proportion of CD4+ lymphocytes in the lavage fluidwas predictive formucosal inflammation (Geboes score $>2$ ) $(\mathrm{p}<0.001)$. IBD ( $\mathrm{UC}$ and $\mathrm{CD})$ patients had a significantly increased $\mathrm{CD} 4+/ \mathrm{CD} 8+$ ratio in the lavage fluid compared to non-IBD patients (6.4 versus $4.3, \mathrm{p}=0.007)$. This increased ratio was found in both active UC patients and patients in remission and therefore could discriminate IBD from non-IBD patients.

Conclusion: An increased CD4 proportion in appendiceal lavage fluid was predictive of a high appendiceal Geboes score in UC patients, and correlated with clinical and immunohistochemical findings in UC, CD and AA patients. In addition, IBD patients show a distinct immunological profile with increased CD4+/CD8+ ratio. If an appendiceal phenotype could be determined predicting clinical response to appendectomy, lavage fluid could be used to identify patients benefitting from resection.
\end{abstract}

Keywords: appendix, inflammatory bowel disease, lymphocytes, inflammation

\author{
Volume 9 Issue 2 - 2018
}

\author{
S Sahami,' TJ Gardenbroek,' JP van Straalen, ${ }^{2}$ \\ MJ van de Vijver, ${ }^{3} \mathrm{M}$ Löwenberg, ${ }^{4} \mathrm{CY}$ \\ Ponsioen, ${ }^{4} \mathrm{GR}$ van den Brink, ${ }^{4} \mathrm{G}$ D'Haens, ${ }^{4}$ \\ WA Bemelman,' CJ Buskens' \\ 'Department of Surgery, Tytgat Institute for Liver and Intestinal \\ Research, Academic Medical Center,The Netherlands \\ ${ }^{2}$ Department of Clinical Chemistry, Tytgat Institute for Liver and \\ Intestinal Research, Academic Medical Center, The Netherlands \\ ${ }^{3}$ Department of Pathology, Tytgat Institute for Liver and \\ Intestinal Research,Academic Medical Center, The Netherlands \\ ${ }^{4}$ Department of Gastroenterology, Tytgat Institute for Liver and \\ Intestinal Research,Academic Medical Center, The Netherlands
}

Correspondence: Christianne Buskens, Department of Surgery, Academic Medical Center, PO Box 22660, I 100 DD Amsterdam, the Netherlands, Tel +3I-20-566 2760, Fax +3I-20566 6569, Email c.j.buskens@amc.uva.nl

Received: February II, 2017 | Published: March 29, 2018

\section{Introduction}

Until recently the appendix was mostly seen as a rudimentary part of the human intestine, but nowadays it has been demonstrated to havea distinct immunological function. Reports are emerging linking this vermiform organ to the development of ulcerative colitis (UC) and a systematic review suggests that an appendectomy could modulate the disease course. ${ }^{1}$ In addition, various animal studies have shown that the removal of the appendix prevents the development of experimental colitis, which further supports this hypothesis. ${ }^{2,3}$

Although the pathogenesis of UC is not fully understood, evidence suggests that the activated immune system is mostly mediated by lymphocytes with a Th2 like phenotype. ${ }^{4}$ Extensive infiltration of subgroups of CD4+ $\mathrm{T}$ cells and elevated cytokine proportions have been observed in the inflamed mucosa of UC patients. ${ }^{5,6}$ Although characteristic transmural histological changes are hardly ever seen in appendectomy specimens of UC patients, a quantitative and qualitative change of the lymphocyte phenotype has been described. A previous study that characterized the histological and immunological characteristics of the appendix in UC patients demonstrated that the various degrees of inflammation were similar to those found in the colon and rectum. ${ }^{7}$

In contrast, for patients with Crohn's disease (CD) higher incidence rates after appendectomy have been described ${ }^{8}$ However, these data are difficult to interpret since the appendix is frequently involved as part of terminal ileitis, which could result in overestimated incidence rates. ${ }^{9-11}$ Most specimens show macroscopically and microscopically affected appendices with transmural inflammation, which is comparable to the affected terminal ileum. Acute appendicitis (AA) represents a different form of transmural inflammation. This nonautoimmune coordinated inflammation has been linked to bacterial invasion, diet, familial aggregation and an obstructing appendiceal faecolith, which possibly play a role in the aetiology of the disease. ${ }^{12-14}$

To gain insight in the distinct role of the appendix in the development of UC, it would be interesting to compare immunological changes between inflammatory and non-inflammatory specimens. The scarce literature on human UC appendices only discusses inflammatory characteristics in resection material. However, If T-cell infiltration and characterization could be clinically determined in the appendix, it might be possible to utilize this as a measurement for the inflammatory process, and guide clinical decision making. Ideally, the immunological phenotype could be used to predict clinical response to appendectomy. During colonoscopy, the orifice of the appendix can be identified which creates the possibility to perform an appendiceal lavage with additional FACs analysis of lymphocyte populations.

The objective of this study was to evaluate if appendiceal lymphocyte infiltration can be determined by analysis of lavage fluid and whether discrimination was possible between UC (remission and active), $\mathrm{CD}$, acute appendicitis, and non-inflammatory controls. 


\section{Materials and methods}

\section{Patient selection}

This prospective cohort study was performed in a tertiary IBD centre between August 2011 and December 2015. UC patients over 18 years of age with therapy refractory UC scheduled for elective colectomy and UC patients in remission participating in the ACCURE trial (a randomized controlled trial analysing the effect of an appendectomy in maintaining remission in UC patients, trial register; NTR2883) were included.(15) The control groups consisted ofpatients with $\mathrm{CD}$ undergoing ileocolic resection, AA patients undergoing laparoscopic appendectomy, and patients undergoing (partial) colectomy for colonic carcinoma or familial adenomatous polyposis (FAP). Demographic data such as gender, age, medication use and details concerning the extent and duration of the disease was registered. Each patient signed an informed consent form approved by the institutional review board.

\section{Clinical disease activity}

In UC patients, disease activity was determined by the full Mayo score measured within 3 months before surgery. ${ }^{16}$ Active disease was defined as a score of $>5$ (with at least an endoscopic subscore of $\geq 2$ ). In CD patients,active disease was defined as inflammation shown onrecent CT and/or MRI imaging and endoscopy. Patients with a stenosis, without inflammation were defined as inactive. In AA patients, only macroscopically affected appendices found during surgery were resected. All control patients were defined as inactive disease.

\section{Surgical procedure}

Surgical procedures were performed by or undersupervision of a dedicated gastrointestinal surgeon. Care was taken not to touch the appendix during dissection. After resection, the specimen was extracted from the abdominal cavity and the appendix was removed from the resection specimen under sterile conditions. The mesentery of the appendix was removed and the appendicular tissue was cleaned of peri-appendicular fat. The distal tip of the appendix was cut off to enable flushing the appendix with fluid. The appendix was inserted in a transparent tube to provide circular pressure during flushing.
Subsequently, the appendix was flushed with $2 \mathrm{cc}$ of phosphate buffered saline (PBS). In case of faecal contamination of the fluid, a second flush with $2 \mathrm{cc}$ of PBS was performed. The lavage fluid was collected in a container with a protein medium and analysed in the clinical chemical laboratory of the AMC. Subsequently, the appendix was transported to the department of pathology for histological evaluation and immunohistochemical staining.

\section{Histology}

Formalin-fixed and paraffin-embedded appendiceal specimens were cut and H\&E-stained in preparation for histological evaluation according to standard operating procedures. Tissue was evaluated by a dedicated gastrointestinal pathologist assessing architectural and inflammatory features. Inflammation was graded according to the validated Geboes scoring system, which subdivides 6 grades based on structural (architectural) change: no abnormality, chronic inflammatory infiltrate, lamina propia neutrophils and eosinophils, neutrophils in epithelium, crypt destruction, and erosions or ulcerations. ${ }^{17}$ Scores can range from 0 to 5 , with higher scores indicating more severe histological inflammation ulceration. Active inflammation was defined as grade 2 or higher. Although the Geboes score hasonlybeen validated for $\mathrm{UC}$, this scoring system was also used for $\mathrm{CD}$ and $\mathrm{AA}$ to grade the severity of inflammation to ensure comparability. No other validated grading scales are available for these conditions.

\section{Immunohistochemistry}

Paraffin embedded slides were stained with CD4 and CD8 antibodies(Biolegend, San Diego, CA) for immunohistochemical analysis. Results were assessed by three reviewers (SS, HH and CB) independently, blinded for the patients' clinical records and disease diagnosis, and scored according to the number of positive cells per high power field. A representative mucosal area was chosen which was not directly covering a lymphoid follicle in the submucosa or lamina propria of the appendiceal wall. Scores were adapted from Stumpf et al. ${ }^{18}$ (grade 1 representing no staining and grade 4 extensive lymphocyte infiltration). ${ }^{18}$ Increased infiltration was defined as a score of $>1$. The cases in which no consensus was reached regarding the grading, a fourth member was asked to evaluate the samples (MV) (Figure 1).

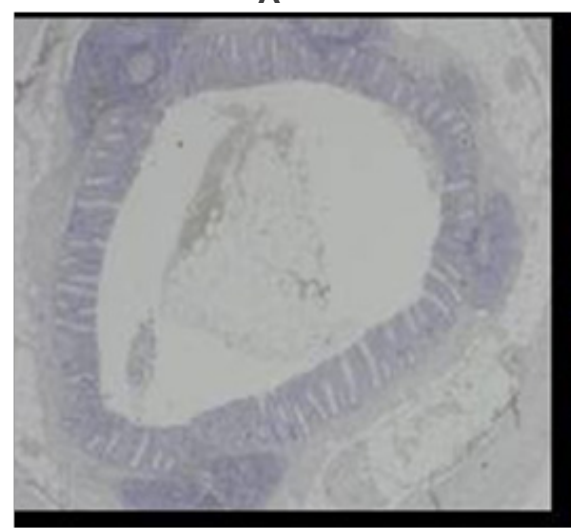

B

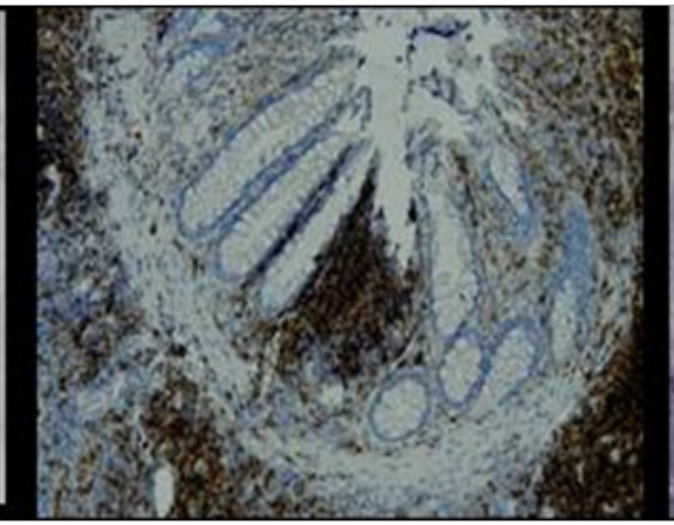

C

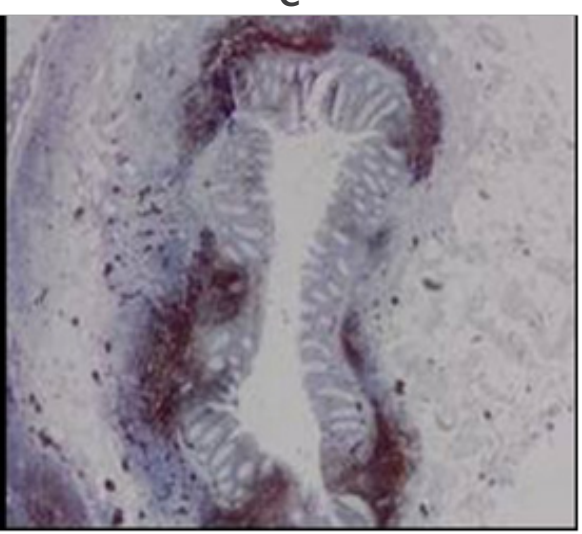

Figure I Immunohistochemical analyses, CD4+ T cell are stained in this image (brown coloured cells). In image A, a normal appendix is shown. The appendix of patients with appendicitis showed extensive influx (grade 3 or 4 ) of CD4+ cells (B).Also in the appendix of UC patients, extensive CD4+ influx can be seen (C). 


\section{Appendiceal lavage fluid analysis}

Mononuclear cells in the lavage fluid were stained with fluorescein is thiocyanate (FITC) and phycoerythrin-conjugated (PE) monoclonal antibodies to $\mathrm{CD} 45+, \mathrm{CD} 3+, \mathrm{CD} 4+$ and CD8+ cells (Biolegend, San Diego, CA). First the phenotyped cells were analysed by flow cytometry (FACS analysis). Cell suspensions were visualised in the forward scatter/side scatter profile, subsequently lymphocytes (CD3+ cells) were gated. The proportion of $\mathrm{CD} 4+$ and $\mathrm{CD} 8+\mathrm{T}$-cells were determined and the relative ratio of $\mathrm{CD} 4+$ and $\mathrm{CD} 8+\mathrm{T}$-cells was calculated (Figure 2).
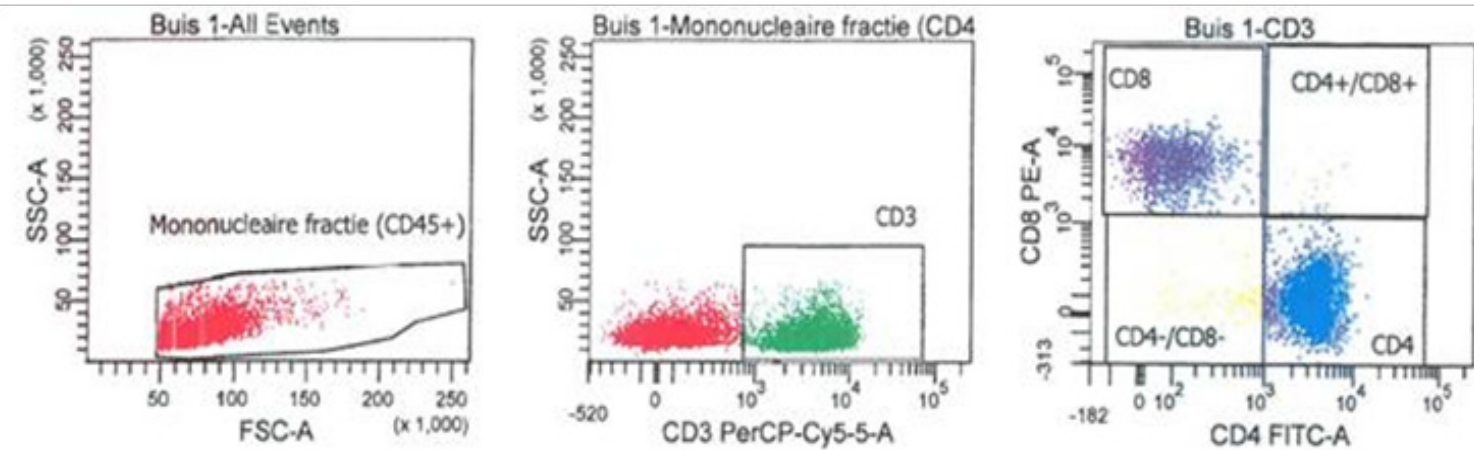

\begin{tabular}{|c|c|c|c|c|}
\hline Population & Parent Name & \#Events. & \%Parent & Population \\
\hline Al Events & \#\#\#\# & 10,000 & \#\#\#\# & All Events \\
\hline Mononucleaire fractie (CD45+) & All Events & 9,998 & 100.0 & Mononucleaire fractie (CD45+) \\
\hline CD3 & Mononucleair... & 6,886 & 68.9 & $\cos$ \\
\hline CD4 & CD3 & 5,261 & 76.4 & $\mathrm{CD} 4$ \\
\hline CD8 & CD3 & 1,246 & 18.1 & CDB \\
\hline CD4-/CD8. & CD3 & 356 & 5.2 & CD4 $4 / C D 8$ \\
\hline $\mathrm{CD} 4+/ \mathrm{CD} 8+$ & CD3 & 30 & 0.4 & $\mathrm{CD} 4+/ \mathrm{CDB}+$ \\
\hline
\end{tabular}

Figure 2 Image of a colour flow cytometry of appendix lavage fluid. Cell suspensions were visualised in the forward scatter/side scatter profile, subsequently lymphocytes were gated. The proportion of CD4+ and CD8+ T cells and the CD4/CD8 ratio in the totally lymphocyte populations were calculated.

\section{Statistical analysis}

Categorical data are presented as frequencies and percentages. Continuous data are presented as mean and standard deviation (SD) or as median and interquartile range (IQR) according to the distribution. Several comparisons were made; UC patients were compared to all other patients groups, active cases (active $\mathrm{UC}, \mathrm{CD}$ and $\mathrm{AA}$ ) were compared to non-active cases (UC in remission and non-inflammatory controls) and IBD cased (UC and CD) were compared to non-IBD cases (AA and non-inflammatory controls).

Independent t-test was used to compare means. Mann-Whitney-U test was used for continuous, not normally distributed data. The Kruskal Wallis test was used when more than 2 groups were compared. To compare dichotomous data the $\chi 2$-test or Fisher's exact test were used. The optimal cut-off value for increased lavage $\mathrm{CD} 4+\mathrm{T}$ cell proportion discriminating between active and inactive disease was determined using receiver operating characteristic (ROC) analysis and determining the highest Youden index. The Youden index is calculated by the following formula: (sensitivity+specificity) -1 . Pearson correlations were used to examine relationships between disease activity, immunohistochemistry and lavage. The following crude estimates are generally used for interpreting strengths of correlations: if $r=0.70$ or higher, there is a very strong relationship; if $\mathrm{r}=0.40$ to 0.6 , there is a strong relationship, if $\mathrm{r}=0.30$ to 0.39 , there is a moderate relationship; if $r=0.20$ to 0.29 , there is a weak relationship and if $r=0.01$ to 0.19 , there is no or negligible relationship. All tests were analysed two-sided and a P-value of $<0.05$ was deemed significant. Statistical analysis was done with IBM SPSS Statistics for Windows ${ }^{\circledR}$, Version 22.0 (IBM Corp., Armonk, NY, United States).

\section{Results}

\section{Demographics}

A total of 41 patients were included; $15 \mathrm{UC}$ patients, $9 \mathrm{CD}$ patients, 7 AA patients and 10 non-inflammatory controls (colonic carcinoma or FAP). Five UC patients were in remission with a median mayo score of 3 (IQR, 3 -4) and 10 UC patient had clinically active disease with a median mayo score of 11 (IQR, 10-11). All 9 CD patients had active disease of which 4 also showed stenosis. The characteristics of the patient groups are summarized in Table 1 .

\section{Histology}

The appendices of all UC patients appeared macroscopically normal, but showed mucosal based inflammation with Geboes score $\geq 2$ in $12 / 15$ patients. In contrast, all CD and AA patients demonstrated macroscopically abnormal appendices with an increased diameter, thickened meso-appendix, and a fibrino-purulent exudate covering the serosa. Histology confirmed the transmural inflammation with oedema and lymphocyte influx present in the macroscopically affected appendices. The appendices of all non-inflammatory controls were both macroscopically and histologically normal. The outcome measures are shown in Table 2. 
Table I Baseline patient characteristics

\begin{tabular}{|c|c|c|c|c|c|}
\hline & \multicolumn{3}{|l|}{ IBD } & \multicolumn{2}{|l|}{ Non-IBD } \\
\hline & UC & & CD & AA & Controls \\
\hline & Remission & Active & & & \\
\hline Totaln ( \%) & 5 & 10 & 9 & 7 & 10 \\
\hline Male & $2(40)$ & $4(40)$ & $2(22)$ & $4(57)$ & $5(50)$ \\
\hline Age at surgery* & $43[26-63]$ & $43[40-54]$ & $25[2|-3|]$ & $43[38-52]$ & $6 \mid[56-70]$ \\
\hline \multicolumn{6}{|l|}{ Extent of UC } \\
\hline Proctitis & $2(40)$ & $2(20)$ & & & \\
\hline Left sided & I (20) & $3(30)$ & & & \\
\hline Pancolitis & $2(40)$ & $5(50)$ & & & \\
\hline \multicolumn{6}{|l|}{ Disease activity } \\
\hline Mayo score* & $3[3-4]$ & $\mathrm{II}[10-1 \mathrm{I}]$ & & & \\
\hline Stenosis & & & $4(44) \dagger$ & & \\
\hline Diseaseduration (months)* & & $66[25-219]$ & 19 [13-93] & NA & $I[0-I]$ \\
\hline \multicolumn{6}{|l|}{ Medication } \\
\hline None & $4(80)$ & $0(0)$ & $\mathrm{I}(\mathrm{II})$ & & \\
\hline Steroids & I (20) & $6(60)$ & $2(22)$ & & \\
\hline Immunomodulators & $0(0)$ & $I(10)$ & $2(22)$ & & \\
\hline Anti-TNF & $0(0)$ & $3(30)$ & $3(33)$ & & \\
\hline Combination & $0(0)$ & $0(0)$ & $\mathrm{I}(\mathrm{II})$ & & \\
\hline
\end{tabular}

Table 2 Outcomemeasures

\begin{tabular}{|c|c|c|c|c|c|}
\hline & \multicolumn{3}{|l|}{ IBD } & \multicolumn{2}{|c|}{ Non-IBD } \\
\hline & \multicolumn{2}{|l|}{ UC } & \multirow[t]{2}{*}{ CD } & \multirow[t]{2}{*}{ AA } & \multirow[t]{2}{*}{ Controls } \\
\hline & Remission & Active & & & \\
\hline Totaln (\%) & 5 & 10 & 9 & 7 & 10 \\
\hline \multicolumn{6}{|l|}{ Histologyn (\%) } \\
\hline Macroscopically inflamed & $0(0)$ & $0(0)$ & $9(100)$ & $7(100)$ & $0(0)$ \\
\hline Microscopically inflamed & $4(80)$ & $8(80)$ & $9(100)$ & $7(100)$ & $0(0)$ \\
\hline \multicolumn{6}{|c|}{ Immunohistochemistry n (\%) } \\
\hline \multicolumn{6}{|l|}{ CD4+ cellcount } \\
\hline Grade I & $3(60)$ & $\mathrm{I}(\mathrm{I0})$ & I (II.I) & I (I4.3) & $7(70)$ \\
\hline Grade 2 & $0(0)$ & $6(60)$ & $2(22.2)$ & $4(57.1)$ & $3(30)$ \\
\hline Grade 3 & $2(40)$ & $3(30)$ & $3(33.3)$ & I (I4.3) & $0(0)$ \\
\hline Grade 4 & $0(0)$ & $0(0)$ & $3(33.3)$ & I (I4.3) & $0(0)$ \\
\hline \multicolumn{6}{|l|}{ CD8+ cellcount } \\
\hline Grade I & I (20) & $\mathrm{I}(10)$ & $2(22.2)$ & I (I4.3) & $\mathrm{I}(\mathrm{I0})$ \\
\hline Grade 2 & $4(80)$ & $7(70)$ & $7(77.8)$ & $5(71.4)$ & $8(80)$ \\
\hline Grade 3 & $0(0)$ & $2(20)$ & $0(0)$ & I (I4.3) & I (I0) \\
\hline Grade 4 & $0(0)$ & $0(0)$ & $0(0)$ & $0(0)$ & $0(0)$ \\
\hline
\end{tabular}




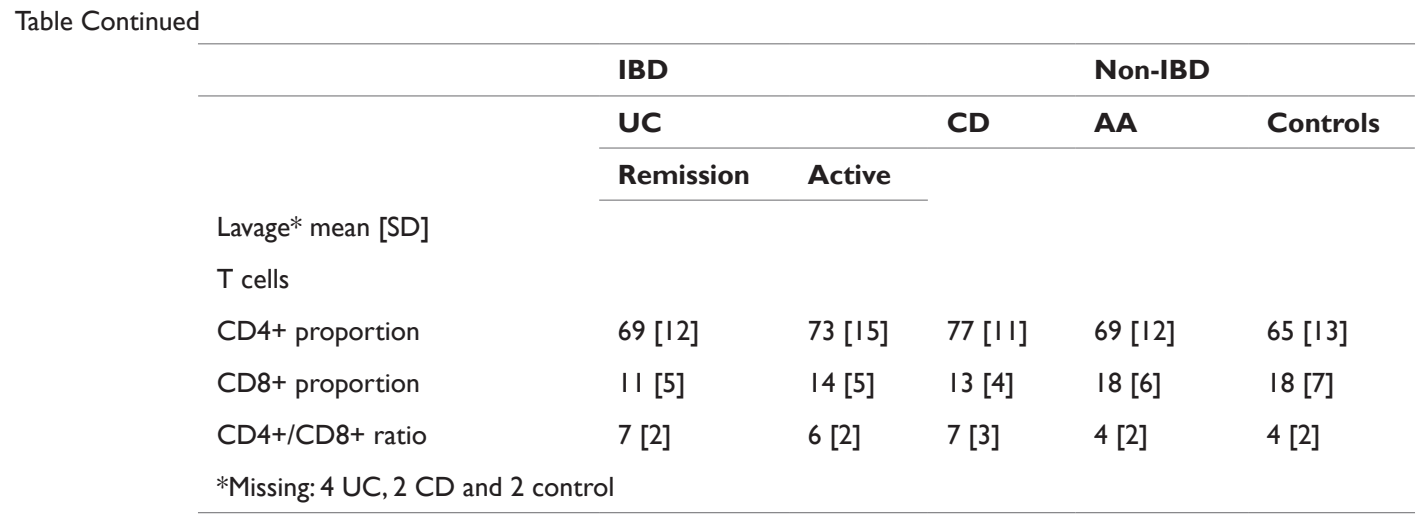

\section{Immunohistochemistry}

Representative examples of immunohistochemical results are presented in Figure 2 (grade 1-4). There was a 91.7\% consensus on discrimination between grade 1 (no increased influx) and grades 2-4 (increased influx). Immunohistochemistry demonstrated that the increased number of lymphocytes seen at histology were predominantly $\mathrm{CD} 4+\mathrm{T}$ cells. The appendices of patients with active disease (active UC, CD and AA) showed a significantly increased influx of mucosal CD4 $+\mathrm{T}$ cells (grade 2-4) with no significant
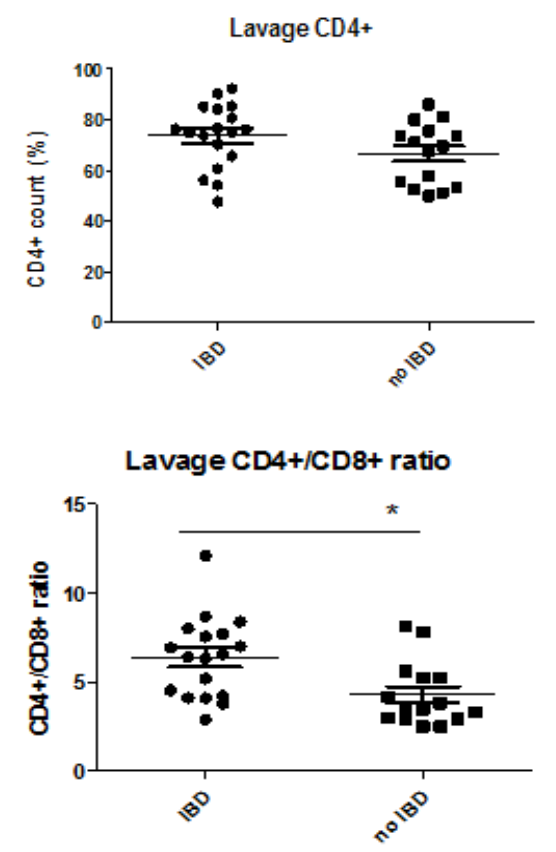

discrepancy between active UC (90\%), CD (89\%), and AA patients $(86 \%)$ (Figure 3). Since increased mucosal CD4+ T cells were only found in $2 / 5 \mathrm{UC}$ patients in remission and $2 / 8$ non-inflammatory controls, this was highly predictive of clinical disease activity $(\mathrm{r}=0.70$, $\mathrm{p}<0.001)$. On immunohistochemistry, no significant differences could be found in the CD8+ lymphocytes between all patient groups, although a relative increase was suggested in AA (Table 2). Due to the categorical histopathological scoring, no meaningful CD4/CD8 ratio could be calculated.
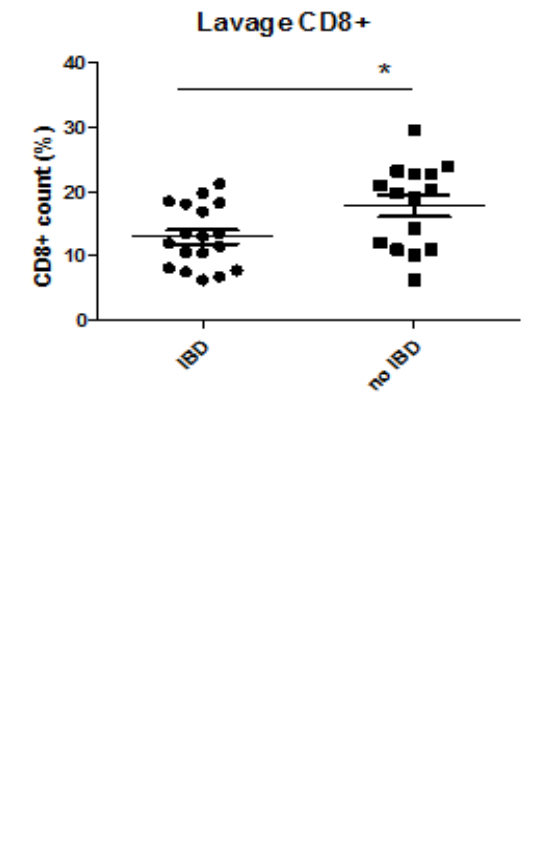

Figure 3 Proportions of CD4+ lymphocytes, CD8+ lymphocytes and CD4/CD8 ratio in the lavage fluid. Comparisons are made between IBD and non-IBD patients. The asterisk indicates a significant difference.

\section{Appendiceal lavage}

In 7 patients ( $3 \mathrm{UC}, 2 \mathrm{CD}$ and 2 control) analysis of the lavage fluid could not be performed due to luminal obliteration or excessive faecal contamination and therefore technical flushing problems. Comparable to immunohistochemical results, the median proportion of CD4+ lymphocytes inthe clinically active cases (active UC, CD, AA) were significantly higher than non-active cases (UC remission and non-inflammatory controls), with no significant difference between the mean proportion of CD4+ lymphocytes between IBD and non-IBD patients ( $p>0.05$ ). The optimal cut-off value to discriminate between active and inactive disease was $>56 \%$ (sensitivity 0.94 and specificity 0.37 ) for CD4+ lymphocytes per lavage. CD4 proportion in the lavage correlated significantly with clinical disease activity $(\mathrm{r}=0.42, \mathrm{p}=0.018)$, with the Geboes score $(\mathrm{r}=0.65, \mathrm{p}=0.012)$, and with CD4+ lymphocyte grading on immunohistochemistry ( $\mathrm{r}=0.43$, $\mathrm{p}=0.033$ ). A high CD4 proportion in lavage was predictive for a high Geboes score (OR 40; $\mathrm{p}=0.002$ ). In the lavage fluid, IBD patients 
(UC and $\mathrm{CD}$ ) had relatively low $\mathrm{CD} 8+$ proportions compared to nonIBD patients (AA and non-inflammatory controls) (17.8 versus 13.0, $\mathrm{p}=0.022$ ) resulting in a significantly increased $\mathrm{CD} 4+/ \mathrm{CD} 8+$ ratio for IBD patients (6.4 versus $4.3, \mathrm{p}=0.007$ ) (Figure 4$)$. This increased ratio was found in both active and non-active UC patients and therefore could discriminate IBD from non-IBD patients.

\section{Discussion}

The link between the appendix and UC has been demonstrated in various epidemiological studies, but the etiological connection remains unknown. The aim of this study was to characterize the T-cell phenotype in appendiceal tissue, correlate findings to clinical disease activity, and analyse if appendiceal lavage fluid was predictive of the immunological profile compared to the gold standard histology. As hypothesized, we found that despite a macroscopically normal appendix, most UC patients havemucosal inflammation and increased numbers of mucosal CD4+ lymphocytes inimmunohistochemical staining of resected appendices. An increased CD4 proportion in appendiceal lavage fluid, was predictive of a high appendiceal Geboes score in UC patients, and correlated with immunohistochemical findings in UC, CD and AA patients. In contrast, increased levels of CD8+lymphocytes were only seen in AA, resulting in an increased CD4+/CD8+ ratio of the lavage fluid of IBD patients. This skewing of $\mathrm{CD} 4+/ \mathrm{CD} 8+$ ratio was seen in patients with both active and remissive $\mathrm{UC}$ and in patients with $\mathrm{CD}$.

Several theories have been proposed that explain the immunomodulating role of the appendix in UC. ${ }^{1}$ One of the theories that has been suggested is that inflammation in the appendix may trigger inflammation in colonic mucosa by releasing inflammatory mediators into the appendiceal lumen. ${ }^{19}$ The appendix is known to be part of the gut-associated lymphoid tissue and is predominantly populated by naive T and B cells. ${ }^{20,21}$ Here, T-lymphocytes are likely to be activated by various luminal antigens and may regulate the immunoglobulin-A (IgA)-producing B cells that home to the colon. ${ }^{22}$ Although it is not clear how a dysbalance of $\operatorname{IgA}$ may predispose to $\mathrm{UC}$, it is interesting to hypothesise that removal of the appendix could mitigate this response. A previous study supports this theory by demonstrating an increased proportion of immature plasma cells in the appendix of UC patients, irrespective of disease activity, suggesting a possible primary role of humoral immune responses in the pathogenesis of UC. In addition, they showed increased numbers of proliferative lymphocytes (Ki-67+CD3+ cells) in the appendix of UC patients compared with $\mathrm{CD}, \mathrm{AA}$ and non-inflammatory control patients, which also indicated involvement of T cell lineage. ${ }^{23}$ Another interesting theory is that the appendix may act as a 'safe house' for commensal bacteria that could repopulatethe gut under certain conditions. This phenomenon has been observed in patients with recurrent Clostridium difficile infections. In UC patients, appendiceal microbiota may cause inflammation due to the deranged mucosal immune system and impaired barrier function. ${ }^{24}$ In this sense, the appendix could presents as a priming site for UC.

A study by Matsushita et al. also demonstrated an increased $\mathrm{CD} 4+/ \mathrm{CD} 8+$ ratio in appendix biopsies of UC patients with active left sided colitis, when compared to non-inflammatory controls. ${ }^{25}$ Interestingly, as the CD4+/CD8+ ratio in the appendix increased, the ratio in the rectum tended to increase as well. These results have led to the suggestion that the CD4+/CD8+ ratio in the mucosa of the appendix represents the severity of inflammation in the colonic mucosa. Although we could not demonstrate a higher CD4+/CD8+ ratio in active disease, the finding of an elevated CD4+/CD8+ ratio in UC patients in remission is intriguing. If indeed this appendiceal immunological dysbalance would contribute to the initiation of UC relapse, this could explain why reduced relapsing rates have been described after appendectomy. ${ }^{26,27}$

Unfortunately, an appendectomy will not be effective in all UC patients. The best results so far have been described by Bolin et al, with $40 \%$ clinical remission after appendectomy. ${ }^{28}$ Appendiceal resection also harbours the risk of surgical complications, and recently a retrospective study suggested that appendectomized UC patients might have an increased risk of colorectal cancer. ${ }^{29}$ Although these results from a small single centre study might have suffered from inclusion bias and results could be influenced by confounders since appendectomized patients had longer follow up with their colon in situ, it also demonstrates that it is of crucial importance to identify patients possibly benefitting from resection, without exposing all UC patients to the possible disadvantages from appendectomy. Therefore, demonstrating an aberrant immunological profile with increased proportion of CD4 lymphocytes in lavage fluid could guide clinical decision making.

The $\mathrm{CD} 4+/ \mathrm{CD} 8+$ ratio has been used as a diagnostic tool in a variety of diseases, including pulmonary, HIV and autoimmune diseases. $^{30-33}$ In these diseases, the $\mathrm{CD} 4+/ \mathrm{CD} 8+$ ratio is used as an indicator for immune dysfunction. An intriguing disease that shows analogy with autoimmune diseases is sarcoidosis, a systemic granulomatous disease of unknown aetiology that primarily affects the lung. The sarcoid reaction is characterized by accumulation of activated $\mathrm{T}$ cells and macrophages at sites of inflammation compared to other interstitial lung diseases such as hypersensitivity pneumonitis in which the lung infiltrates are characterized by cells bearing suppressor/cytotoxic phenotype. ${ }^{34}$ A previous study investigated the clinical usefulness of bronchoalveolar lavage (BAL) cellular analysis with lymphocyte subsets and showed a significant increase in the percentage of CD4+ cells, a decrease in CD8+ cells, and an increase in the $\mathrm{CD} 4+/ \mathrm{CD} 8+$ ratio in patients with sarcoidosis compared to other interstitial lung disease..$^{35}$ The elevated $\mathrm{CD} 4+/ \mathrm{CD} 8+$ ratio in BAL fluid may confirm the diagnosis in patients presenting with a typical picture of sarcoidosis. These findings are in line with our data for IBD.

In this study, we have prospectively identified patients with different inflammatory and non-inflammatory diseases and evaluated their appendiceal tissue. The appendices were evaluated on all levels; macro- and microscopically and by lavage of the appendiceal lumen. Assessment of the histological and immunohistochemical samples was blinded for diagnosis to avoid review bias. The intraand inter-observer measurement error variability was restricted by independently repeating the sample scoring three times and by using three observers.

Unfortunately, the small numbers in every group precluded correlation with varying inflammation grades of disease. Nevertheless, we are the first that have adopted the lavage method on appendices in order to differentiate between diseases of the gastrointestinal tract.

\section{Conclusion}

In conclusion we found that despite a macroscopically normal appearance, appendices of most UC patients show histological 
characteristics of mucosal inflammation, with increased mucosal CD4+ lymphocytes. An increased CD4 proportion in appendiceal lavage fluid, was predictive of a high appendiceal Geboes score in UC patients, and correlated with clinical and immunohistochemical findings in UC, CD and AA patients. In addition, IBD patients show a distinct immunological profile with increased $\mathrm{CD} 4+/ \mathrm{CD} 8+$ ratio. If an appendiceal phenotype could be determined predicting clinical response to appendectomy, lavage fluid could be used to identify patients benefitting from resection.

\section{Author contributions}

S Sahami, TJ Gardenbroek and CJ Buskens designed the research; S Sahami, TJ Gardenbroek and JP van Straalenperformed the research; S Sahami, CJ Buskens, TJ Gardenbroek, MJ van de Vijver graded the histological slides, S Sahami, TJ Gardenbroek, M Löwenberg, CY Ponsioen, GR van den Brink, G D'Haens, WA Bemelman, CJ Buskens analysed the data; S Sahami, TJ Gardenbroek and CJ Buskens wrote the pap

\section{Acknowledgements}

Non

\section{Conflicts of interest and source of funding}

There were no conflicts of interest, funding or support for this study.

\section{References}

1. Sahami S, Kooij IA, Meijer SL, et al. The Link between the Appendix and Ulcerative Colitis: Clinical Relevance and Potential. Am J Gastroenterol. 2016;111(2):163-9.

2. Krieglstein CF. Role of Appendix and Spleen in Experimental Colitis . $J$ Surg Res. 2001;101(2):166-75.

3. Farkas SA, Hornung M, Sattler C, et al. Preferential Migration of CD62L + Cells into the Appendix in Mice with Experimental Chronic Colitis. Eur Surg Res. 2005;37(2):115-22.

4. Heller F, Florian P, Bojarski C, et al. Interleukin-13 Is the Key Effector Th2 Cytokine in Ulcerative Colitis That Affects Epithelial Tight Junctions, Apoptosis, and Cell Restitution. Gastroenterology. 2005;129(2):550-64.

5. Fujino S, Andoh a, Bamba S, et al. Increased expression of interleukin 17 in inflammatory bowel disease. Gut. 2003;52(1):65-70.

6. Abraham C, Cho JH. Inflammatory bowel disease. $N$ Engl $\mathrm{J}$ Med. 2009;361:2066-78.

7. Jo Y, Matsumoto T, Yada S, et al. Histological and immunological features of appendix in patients with ulcerative colitis. Dig Dis Sci. 2003;48(1):99-108.

8. Kaplan GG, Pedersen BV, Andersson RE, et al. The risk of developing Crohn's disease after an appendectomy: a population-based cohort study in Sweden and Denmark. Gut. 2007;56(10):1387-92.

9. Haddad M, Azim F, Koren A, et al. Crohn's disease of the appendix. Eur J Surg. 1993;159(3):191-2.

10. Prieto-Nieto I, Perez-Robledo JP, Hardisson D, et al. Crohn's disease limited to the appendix. Am J Surg. 2001;182(5):531-3.

11. Vanek VW, Spirtos G, Awad M, et al. Isolated Crohn's disease of the appendix. Two case reports and a review of the literature. Arch Surg. 1988;123(1):85-7
12. Basta M, Morton NE, Mulvihill JJ, et al. Inheritance of acute appendicitis: familial aggregation and evidence of polygenic transmission. Am J Hum Genet. 1990;46(2):377-82.

13. Jones BA, Demetriades D, Segal I, et al. The prevalence of appendiceal fecaliths in patients with and without appendicitis. A comparative study from Canada and South Africa. Ann Surg. 1985;202(1):80-2.

14. Walker AR, Segal I. What causes appendicitis? J Clin Gastroenterol. 1990;12(2):127-9.

15. Gardenbroek TJ, Pinkney TD, Sahami S, et al. The ACCUREtrial: the effect of appendectomy on the clinical course of ulcerative colitis, a randomised international multicenter trial (NTR2883) and the ACCURE-UK trial: a randomised external pilot trial (ISRCTN56523019). BMC Surg. 2015;15:30.

16. Lewis JD, Chuai S, Nessel L, et al. Use of the noninvasive components of the Mayo score to assess clinical response in ulcerative colitis. Inflamm Bowel Dis. 2008;14(12):1660-6.

17. Geboes K, Riddell R, Ost a, et al. A reproducible grading scale for histological assessment of inflammation in ulcerative colitis. Gut. 2000;47(3):404-9.

18. Stumpf M, Hasenburg A, Riener M-O, et al. Intraepithelial CD8positive $\mathrm{T}$ lymphocytes predict survival for patients with serous stage III ovarian carcinomas: relevance of clonal selection of T lymphocytes. Br J Cancer. 2009;101(9):1513-21.

19. Kawanishi H. Immunocompetence of normal human appendiceal lymphoid cells: in vitro studies. Immunology. 1987;60(1):19-28.

20. Spencer J, Finn T, Isaacson PG. Gut associated lymphoid tissue: a morphological and immunocytochemical study of the human appendix. Gut. 1985;26(7):672-9.

21. Kawanishi H. Immunocompetence of normal human appendiceal lymphoid cells: in vitro studies. Immunology. 1987;60(1):19-28.

22. Masahata K, Umemoto E, Kayama H, et al. Generation of colonic IgAsecreting cells in the caecal patch. Nat Commun. 2014;5:3704.

23. Kawachiya T, Oshitani N, Jinno Y, et al. Significance of increased proliferation of immature plasma cells in the appendix of patients with ulcerative colitis. Int J Mol Med. 2005;15(3):417-23.

24. Kooij IA, Sahami S, Meijer SL, et al. The immunology of the vermiform appendix: a review of the literature. Clin Exp Immunol. 2016;186(1):1-9.

25. Matsushita M, Uchida K, Okazaki K. Role of the appendix in the pathogenesis of ulcerative colitis. Inflammopharmacology. 2007;15(4):154-7.

26. Naganuma M, Iizuka B, Torii A, et al. Appendectomy Protects Against the Development of Ulcerative Colitis and Reduces Its Recurrence : Results of a Multicenter Case-Controlled Study in Japan. Am J Gastroenterol. 2001;96(4):1-4.

27. Cosnes J, Carbonnel F, Beaugerie L, et al. Effects of appendicectomy on the course of ulcerative colitis. Gut. 2002;51(6):803-7.

28. Bolin TD, Wong S, Crouch R, et al. Appendicectomy as a therapy for ulcerative proctitis. Am J Gastroenterol. 2009;104(10):2476-82.

29. Parian A, Limketkai B, Koh J, et al. Appendectomy does not decrease the risk of future colectomy in UC: results from a large cohort and meta-analysis. Gut. 2017;66(8):1390-1397.

30. Danila E, Norkūnienè J, Jurgauskienè L, et al. Diagnostic role of BAL fluid CD4/CD8 ratio in different radiographic and clinical forms of pulmonary sarcoidosis. Clin Respir J. 2009;3(4):214-21. 
31. Serrano-Villar S, Sainz T, Lee SA, et al. HIV-infected individuals with low $\mathrm{CD} 4 / \mathrm{CD} 8$ ratio despite effective antiretroviral therapy exhibit altered $\mathrm{T}$ cell subsets, heightened $\mathrm{CD} 8+\mathrm{T}$ cell activation, and increased risk of non-AIDS morbidity and mortality. PLoS Pathog. 2014;10(5):e1004078.

32. Yin Y, Qin J, Dai Y, et al. The CD4+/CD8+ Ratio in Pulmonary Tuberculosis: Systematic and Meta-Analysis Article. Iran J Public Health. 2015;44(2):185-93.

33. Taubert R, Hardtke-Wolenski M, Noyan F, et al. Intrahepatic regulatory $\mathrm{T}$ cells in autoimmune hepatitis are associated with treatment response and depleted with current therapies. J Hepatol. 2014;61(5):1106-14.
34. Semenzato G. Immunology of interstitial lung diseases : cellular events taking place in the lung of sarcoidosis, hypersensitivity pneumonitis and HIV infection. Eur Respir J. 1991;4(1):94-102.

35. Lee W, Chung WS, Hong K, et al. Clinical Usefulness of Bronchoalveolar Lavage Cellular Analysis and Lymphocyte Subsets in Diffuse Interstitial Lung Diseases. Ann Lab Med. 2015;35(2):220-5. 